The Journal of Nonlinear $\mathbf{S}_{\text {ciences and }}$ Applications

http://www.tjnsa.com

\title{
ON SOME NEW EMBEDDING THEOREMS FOR SOME ANALYTIC CLASSES IN THE UNIT BALL
}

\author{
ROMI SHAMOYAN ${ }^{1}$ AND MEHDI RADNIA ${ }^{2}$
}

ABSTRACT. We provide new sharp embedding theorems for analytic classes in unit ball expanding at the same time some previously known assertions.

\section{INTRODUCTION AND NOTATIONS}

Let $B=\left\{z \in C^{n}:|z|<1\right\}$ be the open unit ball of $C^{n}$ and $\mathrm{S}$ the unit sphere of $C^{n}$. Let $d v$ be the normalized Lebesgue measure on $B$ and $d \sigma$ the normalized rotation invariant Lebesgue measure on $S$. We denote by $H(B)$ the class of all holomorphic functions on $B$. For any real parameter $\alpha$ we consider the weighted volume measure

$$
d v_{\alpha}(z)=\left(1-|z|^{2}\right)^{\alpha} d v(z)
$$

It is well known that $v_{\alpha}$ is a finite measure if and only if $\alpha>-1$. Suppose $0<p<\infty$ and $\alpha>-1$, the weighted Bergman space $A_{\alpha}^{p}$ consists of those functions $f \in H(B)$ for which

$$
\|f\|_{A_{\alpha}^{p}}^{p}=\int_{B}|f(z)|^{p} d v_{\alpha}(z)<\infty .
$$

When $\alpha=0$, we get the classical Bergman space, which will be denoted by $A^{p}$. See [8] and [11] for some basic facts on Bergman spaces. Let further $H^{p}(B)$ be the well known Hardy spaces in the unit ball and let further $P[g]$ be the Poisson integral of a function $g \in L^{p}(S)$. Let $\left\{a_{k}\right\}_{k=1}^{\infty}$ be the sampling sequence in the unit ball (see[11]).

Let $r>0$ and $z \in B$, the Bergman metric ball at $\mathrm{z}$ is defined as

$$
D(z, r)=\left\{w \in B: \beta(z, w)=\frac{1}{2} \log \frac{1+\left|\varphi_{z}(w)\right|}{1-\left|\varphi_{z}(w)\right|}<r\right\} .
$$

2000 Mathematics Subject Classification. Primary 32A10; Secondary 32A37.

Key words and phrases. Area operator, Bergman metric, Bergman metric ball, Carleson measure, Hardy class, nonisotropic ball. 
Here the involution $\varphi_{z}$ has the form

$$
\varphi_{z}(w)=\frac{z-P_{z} w-s_{z} Q_{z} w}{1-<w, z>}
$$

where $s_{z}=\left(1-|z|^{2}\right)^{\frac{1}{2}}, P_{z}$ is the orthogonal projection into the space spanned by $z \in B$, i.e., $P_{z} w=\frac{\langle w, z>z}{|z|^{2}}, P_{0} w=0$ and $Q_{z}=I-P_{z}$ (see [11]). The volume of $D(z, r)$ is given by (see [8], [11])

$$
v(D(z, r))=\frac{R^{2 n}\left(1-|z|^{2}\right)^{n+1}}{\left(1-R^{2}|z|^{2}\right)^{n+1}},
$$

where $R=\tanh (r)$. Set $|D(z, r)|=v(D(z, r))$. For $w \in D(z, r), r>0$, we have that (see, for example, [11])

and

$$
\left(1-|z|^{2}\right)^{n+1} \asymp\left(1-|w|^{2}\right)^{n+1} \asymp|1-<z, w>|^{n+1} \asymp|D(z, r)|
$$

For any $\zeta \in S$ and $r>0$, the nonisotrpic metric ball $Q_{r}(\zeta)$ is defined by(see [11])

$$
Q_{r}(\zeta)=\left\{z \in B:|1-<z, \zeta>|^{1 / 2}<r\right\} .
$$

A positive Borel measure $\mu$ on $B$ is called a $\gamma$-Carleson measure if there exists a constant $C>0$ such that

$$
\mu\left(Q_{r}(\zeta)\right) \leq C r^{2 \gamma}
$$

for all $\zeta \in S$ and $r>0$. A well-known result about the $\gamma$-Carleson measure(see [11]) is that $\mu$ is a $\gamma$-Carleson measure if and only if

$$
\sup _{a \in B} \int_{B}\left(\frac{1-|a|^{2}}{|1-<z, a>|^{2}}\right)^{\gamma} d \mu(z)<\infty .
$$

Let $D=\{z:|z|<1\}, T=\{z:|z|=1\}$ and $\partial D$ the boundary of $D$. For any $\xi \in \partial D$, the cone $\Gamma_{\gamma}(\xi)$ on $\mathrm{D}$ is defined by

$$
\Gamma_{\gamma}(\xi)=\{z \in D:|1-\bar{\xi} z|<\gamma(1-|z|)\}, \quad \forall \xi \in \partial D \quad \text { and } \quad \gamma>1 .
$$

Let $\mu$ be a nonnegative measure on $D$. The area operator on the unit disk was defined by(see, e.g. [4], [10])

$$
A_{\mu}(f)(\zeta)=\int_{\Gamma(\zeta)}|f(z)| \frac{d \mu(z)}{1-|z|} .
$$

The area operator relates to the nontangential maximal function, Littlewood-Paley operator, multipliers and tent space. It is very useful in the harmonic analysis. On the unit disk, the boundedness and compactness of the area operators was studied by Cohn and Wu respectively on the Hardy space and the weighted Bergman space(see [4], [5], [10]). 
Motivated by results of [4], [10], we define the area operator on the unit ball as follows. Let $\mu$ be a positive Borel measure on $B$, we define

$$
G_{\mu, \gamma}(f)(\xi)=\int_{\Gamma_{\gamma}(\xi)} \frac{|f(z)| d \mu(z)}{(1-|z|)^{n}} .
$$

Here for $\gamma>1, \Gamma_{\gamma}(\xi)$ is the corresponding approach region with vertex $\xi$ on $S$, i.e.

$$
\Gamma_{\gamma}(\xi)=\left\{z \in B,|1-<z, \xi>|<\gamma\left(1-|z|^{2}\right)\right\} .
$$

One of the purposes of this paper is to study the area operator on the Hardy space in the unit ball in $C^{n}$. Various emebdding theorems in the unit ball for various analytic classes were proved before by many authors (see ,for example, [2], [3], [4], [6], [7], [9], [11] and references there). Throughout this paper, constants are denoted by $C$, they are positive and may differ from one occurrence to the other. The notation $A \asymp B$ means that there is a positive constant $C$ such that $C^{-1} B \leq A \leq C B$.

\section{MAin RESUlts}

To state and prove our results, let's collect some nice properties of the Bergman metric ball that will be used in this paper.

Lemma 1.([11])There exists a positive integer $N$ such that for any $0<r \leq 1$ we can find a sequence $\left\{a_{k}\right\}$ in $B$ with the following properties:

(1) $B=\cup_{k} D\left(a_{k}, r\right)$;

(2) The sets $D\left(a_{k}, r / 4\right)$ are mutually disjoint;

(3) Each point $z \in B$ belongs to at most $N$ of the sets $D\left(a_{k}, 2 r\right)$.

Remark 1. If $\left\{a_{k}\right\}$ is a sequence from Lemma 1, according to the result on page 76 of [11], there exist positive constants $C_{1}, C_{2}$ such that

$$
C_{1} \int_{B}|f(z)|^{p} d v_{\alpha}(z) \leq \sum_{k=1}^{\infty}\left|f\left(a_{k}\right)\right|^{p}\left(1-\left|a_{k}\right|^{2}\right)^{n+1+\alpha} \leq C_{2} \int_{B}|f(z)|^{p} d v_{\alpha}(z)
$$

Such a sequence will be called a sampling sequence.

Lemma 2.([11]) For each $r>0$ there exists a positive constant $C_{r}$ such that

$$
C_{r}^{-1} \leq \frac{1-|a|^{2}}{1-|z|^{2}} \leq C_{r}, \quad C_{r}^{-1} \leq \frac{1-|a|^{2}}{|1-<z, a>|} \leq C_{r},
$$

for all a and $\mathrm{z}$ such that $\beta(a, z)<r$.

Lemma 3. ([11]) Suppose $r>0, p>0$ and $\alpha>-1$. Then there exists a constant $C>0$ such that

$$
|f(z)|^{p} \leq \frac{C}{\left(1-|z|^{2}\right)^{n+1+\alpha}} \int_{D(z, r)}|f(w)|^{p} d v_{\alpha}(w)
$$


for all $f \in H(B)$ and $z \in B$.

If $H(D)$ is the space of all holomorphic functions in $\mathrm{D}$ and $X \subset H(D)$ is a normed subspace and $\mu$ is a positive Borel measure, then (see [4], [10]) we have a natural problem. Describe all positive Borel measures such that

$$
\int_{T}\left(\int_{\Gamma_{t}(\xi)} \frac{|f(z)|}{(1-|z|)} d \mu(z)\right)^{p} d \sigma(\xi) \leq C\|f\|_{X}^{p} \quad \text { for } 0<p<\infty .
$$

For example (6) is true if and only if $\mu$ is a Carleson measure and if $X=H^{p}, 0<p<\infty$ (see [4]).

Problem. Find (6) type embedding in unit ball and multyfunctional case.

Theorem 2.1. Let $\frac{1}{p}+\frac{1}{q}=1, m>1, m \in N ;\left(\sum_{k=1}^{n} \frac{1}{p_{k}}\right)+\frac{1}{q}=1$. Then let $\mu$ be a positive Borel measure on $B$. Then $\mu$ is a Carleson measure if and only if

$$
\int_{S}\left(\int_{\Gamma_{t}(\xi)} \frac{\prod_{k=1}^{n}\left|f_{k}(z)\right|}{(1-|z|)^{n}} d \mu(z)\right)^{p} d \sigma(\xi) \leq C\left(\prod_{k=1}^{n}\left\|f_{k}\right\|_{H^{p_{k}}}\right),
$$

$f_{k} \in H(B)$ and $k=1, \ldots n$.

Proof. We have $k=1, \ldots, n, f_{k} \in H(B)$ then for $p>1$

$$
K_{1}=\int_{S}\left(\int_{\Gamma_{t}(\xi)} \frac{\prod_{k=1}^{n}\left|f_{k}(z)\right|}{(1-|z|)^{n}} d \mu(z)\right)^{p} d \sigma(\xi)
$$

Let $g \in L^{q}(S)$ and , hence

$$
K_{1}=\int_{S} g(z) \int_{\Gamma_{t}(\xi)} \frac{|\tilde{f}(z)| d \mu(z)}{(1-|z|)^{n}} d \sigma(\xi) .
$$

By use of Fubini theorem we have

Then we have (see [11])

$$
\begin{aligned}
& K_{1}=\int_{B}|\tilde{f}(z)|\left(\frac{1}{(1-|z|)^{n}} \int_{S} \chi_{\Gamma_{\sigma(\xi)}} g(z) d \sigma(\xi)\right) d \mu(z) \\
= & \int_{B}|\widetilde{f}(z)||K g(z)| d \mu(z) .
\end{aligned}
$$

$$
|K g(z)| \leq C P[g](z)
$$

Then we have

$$
K_{1} \leq \int_{B}|\widetilde{f}(z)||P[g](z)| d \mu(z) .
$$


Hence using Holder inequality for $\left(\sum_{k=1}^{n} \frac{1}{p_{k}}\right)+\frac{1}{q}=1$ we will have

$$
K_{1} \leq C\|g\|_{L^{q}(S)} \cdot\left(\prod_{k=1}^{n}\left\|f_{k}\right\|_{H^{p_{k}}}\right) .
$$

Since $\mu$ is a Carleson measure(see [11]).

The reverce follows from estimates for standart test function in the unit ball(see [11]) and modification of Cohn's argument from [5]. Let

$$
\left|f_{j}(z)\right|=\left(\left|1-<z, w_{j}>\right|^{\frac{-m n}{p}}\right),
$$

Such that $w_{j}=\left(1-r_{j}\right) \xi, 0<r_{j}<1, \xi \in S$ then $\|f\|_{H^{p}}^{p} \asymp r_{j}^{-(m-1) n}$.

Hence if $\left[K\left(f_{1}, \ldots, f_{n}\right)\right] \leq C \prod_{k=1}^{n}\left\|f_{k}\right\|_{H^{p_{k}}}$ is true, where this inequality is the main estimate in this theorem, then for $\widetilde{r}=r_{1} \ldots r_{m}, r_{j}=r^{\frac{1}{m}}$ and $r \in(0,1)$ we have

$$
\int_{S}\left(\int_{Q_{\tilde{r}}(\xi) \cap \Gamma_{\sigma}(\widetilde{\xi})} \frac{d \mu(z) d \sigma(\widetilde{\xi})}{\left(r_{1} \ldots r_{m}\right)^{n}(1-|z|)^{n}}\right) \leq C .
$$

By using Cohn's argument in higher dimension and the obvious multyplicative property of two characteristic functions of sets for unit ball we have

$$
\left(\frac{1}{r_{1} \ldots r_{m}}\right) \int_{B}\left(\chi_{Q_{r_{1} \ldots r_{m}(\xi)}}(z)\right) \frac{d \mu(z)}{(1-|z|)^{n}} \times\left(\int_{S} \chi_{\Gamma_{\sigma}(\widetilde{\xi})}(z) d \sigma(\widetilde{\xi}) \leq C .\right.
$$

If we repeat this procedure with $w_{j}=\left(1-r_{j}^{2}\right) \xi$, then we will have

$$
\frac{\mu\left(Q_{\widetilde{r}}(\xi)\right)}{\left(r_{1}^{2} \ldots r_{m}^{2}\right)^{n}} \leq C
$$

Hence $\mu$ is Carleson measure. Theorem 2.1 is proved.

Theorem 2.2. Let $\left\{a_{k}\right\}_{k=1}^{\infty}$ be sampling sequence in the unit ball and $f \in H(B)$. Let $q \in(0, \infty), p \in(0, \infty), r \in(0, \infty)$, let $\mu$ be positive Borel measure on $B, \alpha+\beta>-1, \beta>0$, $\alpha>-1$ then

$$
\int_{B}\left(\int_{D(z, r)}|f(w)|^{p} d \mu(w)\right)^{\frac{q}{p}} d v_{\alpha}(z) \leq C \int_{B}|f(z)|^{q}(1-|z|)^{\alpha+\beta} d v(z)
$$

if and only if

$$
\left(\sup _{a_{k}}\right)\left(\mu\left(D\left(a_{k}, R\right)\right)\right)\left(1-\left|a_{k}\right|\right)^{-\beta \frac{p}{q}}<\infty, \quad \text { forsome } R>0 \quad \text { and forall } k \in N
$$

Proof. The main idea is that for all $r \in(0, \infty)$ and $z \in B$ there exists a decomposition of unit ball $B$ into Bergman metric balls $D\left(a_{k}, R\right)$ for $r \in(0, \infty)$ such that $D(z, r)$ is in the union of some $D\left(a_{k}, R\right)$ balls and the amount of these balls are less than $N$ where $N$ depends only on $n$ (see [11]). This allows us to replace for some $R>0$, by any $R>0$ in formulation of theorem 2.2 (see [10]). Moreover $\beta(z, w)$ is metric(see [11]), hence we can apply known triangle inequality for metric, hence we will have $D(z, r) \subset D\left(a_{k}, r_{1}\right)$ for some $r_{1}>0$ if only 
$z \in D\left(a_{k}, R\right)$ for some $R>0$. Then using preliminary lemmas we formulated above (1) and (2) we have

$$
\begin{gathered}
\int_{B}\left(\int_{D(z, r)}|f(w)|^{p} d \mu(w)\right)^{\frac{q}{p}} d v_{\alpha}(z) \leq \\
C \sum_{k} \int_{D\left(a_{k}, R\right)}\left(\int_{D\left(a_{k}, \widetilde{r}\right)}|f(w)|^{p} d \mu(w)\right)^{\frac{q}{p}} d v_{\alpha}(z) \leq \\
C \sum_{k}\left(\int_{D\left(a_{k}, \widetilde{r}\right)}|f(z)|^{p}(1-|z|)^{\frac{p}{q}(n+1+\alpha)} d \mu(z)\right)^{\frac{q}{p}} \leq \\
C \sum_{k}\left(\max _{D\left(a_{k}, \widetilde{r}\right)}|f(w)|^{q}\right)\left(\left(1-\left|a_{k}\right|\right)^{n+1+\alpha}\right) \times\left(\mu\left(D\left(a_{k}, \widetilde{r}\right)\right)^{\frac{q}{p}} \leq\right. \\
C \sum_{k}\left(\int_{D\left(a_{k}, 2 \widetilde{r}\right)}|f(w)|^{q}(1-|w|)^{\alpha+\beta} d v(w)=\|f\|_{A_{\alpha+\beta}^{q}}^{q} .\right.
\end{gathered}
$$

To show the reverce implication we have to put standard test function $f(w)=\left(\frac{1}{(1-w z)^{\gamma}}\right)$ that $w, z \in B$, for large enough $\gamma>0$ into (9) and apply all standart arguments for estimates of test functions(see [11] theorem 2.25), using simple properities of Bergman metric ball that we indicated in introduction and lemmas 1-3. And estimate

$$
\int_{D\left(a_{k}, \widetilde{r}\right)}\left(\int_{D\left(a_{k}, R\right)}|f(w)|^{p} d \mu(w)\right)^{\frac{q}{p}} d v_{\alpha}(z) \leq C \int_{B}\left(\int_{D(z, r)}|f(w)|^{p} d \mu(w)\right)^{\frac{q}{p}} d v_{\alpha}(z),
$$

for some $\widetilde{r}, R>0$.

The proof of the following theorem is based on same ideas and we omit it.

Theorem 2.3. Let $f \in H(B)$ and $r \in(0, \infty)$, let $\mu$ be positive Borel measure on $B$, $0<p, q<\infty, p \leq q, \beta>0, \alpha>-1, \alpha+\beta \frac{p}{q}>-1$ and let $\left\{a_{k}\right\}_{k=1}^{\infty}$ be a sampling sequence in ball. Then

$$
\begin{gathered}
\left(\int_{B}\left(\int_{D(z, r)}|f(w)|^{p} d v_{\alpha}(w)\right)^{\frac{q}{p}} d \mu(z) \leq\right. \\
C \sum_{k}\left[\left(\int_{D\left(a_{k}, \widetilde{r}\right)}|f(w)|^{p}(1-|w|)^{\alpha+\beta \frac{p}{q}} d v(w)\right)\right]^{\frac{q}{p}} \quad \text { forsome } \quad \widetilde{r}>0
\end{gathered}
$$

if and only if

$$
\mu\left(D\left(a_{k}, R\right)\right) \leq C\left(1-\left|a_{k}\right|\right)^{\beta}, \text { forsome } R>0 \text { and forall } k \in N
$$

Remark 2. The right side of (9) and (10) in theorem 2.2 and 2.3 can be changed with the almost the same proof to $\int_{B}\left(\int_{D(w, r)}|f(z)|^{s} d v(z)\right)^{\frac{t}{s}} d v(w)$ for some $t, s \in(0, \infty)$. 
Theorem 2.4. Let $f \in H(B), 0<p<\infty, \alpha>-1$

(1) $\int_{B}|f(w)|^{p} \mu(D(w, r)) d v_{\alpha}(w) \leq C\|f\|_{A_{\alpha}^{p}, r>0}$ if and only if $\mu\left(D\left(a_{k}, R\right)\right) \leq$ Const for all $k \in N$ and for some $R>0$.

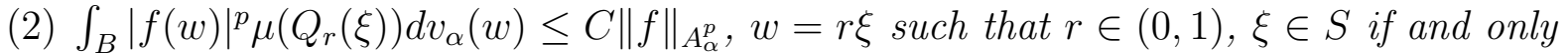
if $\mu\left(D\left(a_{k}, R\right)\right) \leq$ Const for all $k \in N$ and for some $R>0$.

Proof. Let us prove the first part using lemma 1. Indeed using arguments we provided in the proof of theorem 2.2

$$
\begin{gathered}
\int_{B}|f(w)|^{p} \mu(D(w, r)) d v_{\alpha}(w)=\sum_{k} \int_{D\left(a_{k}, R\right)}|f(w)|^{p} \mu(D(w, r)) d v_{\alpha}(w) \\
\mu(D(w, r)) \leq C\left(\int_{D\left(a_{k}, \bar{R}\right)} d \mu(z)+\sum_{s=1}^{N} \int_{D\left(a_{k_{s}}, \bar{R}\right)} d \mu(z)\right) \leq C \int_{D\left(a_{k}, \bar{R}\right)} d \mu(z)+C(N),
\end{gathered}
$$

where $w \in D\left(a_{k}, R\right)$ and $N$ depends only on $n$. Hence

$$
\begin{gathered}
\sum_{k \geq 0} \int_{D\left(a_{k}, R\right)}|f(w)|^{p} \mu(D(w, r)) d v_{\alpha}(w) \leq C \sum_{k \geq 0} \int_{D\left(a_{k}, R\right)}|f(w)|^{p} d v_{\alpha}(w) \\
\leq C\|f\|_{A_{\alpha}^{p}}^{p}, \quad \text { for } 0<p<\infty .
\end{gathered}
$$

To set the reverce we note that if $f(z)=\frac{1}{\left(1-a_{k} z\right)^{\beta}}$, where $\beta>0$ and $\beta$ is large enough. Then we will have from lemma 2

$$
\|f\|_{A_{\alpha}^{p}} \leq \frac{C}{\left(1-\left|a_{k}\right|\right)^{\beta+\frac{-n-1-\alpha}{p}}} .
$$

And it is easy to see for some $R$ and $\widetilde{r}$ there is an estimate

$$
\int_{D\left(a_{k}, R\right)}|f(w)|^{p} \mu\left(D\left(a_{k}, \widetilde{r}\right)\right) d v_{\alpha}(w) \leq C \int_{B}|f(w)|^{p} \mu(D(w, r)) d v_{\alpha}(w) .
$$

The proof of the first part is completed. The proof of the second part follows from small modifications of arguments we provided in the proof of first part of this theorem, and Lemma 5.23 of [11] which states $D(a, R) \subset Q_{r}(\xi), R>0, r \in(0,1), \xi \in S, a=\left(1-v r^{2}\right) \xi, v \in(0,1)$.

\section{REFERENCES}

[1] A. B. Alexandrov, Function Theory in the Ball, in Several Complex Variables II, Springer Verlag, New York, 1994.

[2] C. Cascante and J. Ortega, Carleson measures on spaces of Hardy-Sobolev type, Canad. J. Math. 47 (6) (1995), 1177-1200.

[3] C. Cascante and J. Ortega, On q-Carleson measures for spaces of M-harmonic functions, Canad. J. Math. 49 (4)(1997), 653-674.

[4] C. Cascante and J. Ortega, Imbedding potentials in tent spaces, J. Funct. Anal. 198 (1) (2003), $106-141$.

[5] W. S. Cohn, Generalized area operators on Hardy spaces, J Math Anal Appl. 216 (1)(1997), 112-121. 
[6] J. Ortega and J. Fàbrega, Hardys inequality and embeddings in holomorphic Triebel-Lizorkin spaces, Illinois J. Math. 43 (4)(1999), 733-751.

[7] J. Ortega and J. Fàbrega, Holomorphic Triebel-Lizorkin spaces, J. Funct. Anal. 151 (1)(1997), 177-212.

[8] W. Rudin, Function Theory in the Unit Ball of $C^{n}$, Springer-Verlag, New York, 1980.

[9] R. Shamoyan and O. Mihic, On some properties of holomorphic spaces based on Bergman metric ball and Luzin area operator, J. Nonlinear Sci. Appl. 2 (3)(2009), 183-194

[10] Z. Wu, Area operators on Bergman space, Sci.China Series A. 36(5) (2006), 481-507.

[11] K. Zhu, Spaces of Holomorphic Functions in the Unit Ball, Springer Verlag, New York, 2005.

1 Department of Mathematics, Erevan State University , Armenia.

E-mail address: rshamoyan@yahoo.com

2 Department of Mathematics, Tabriz University, Tabriz, Iran.

E-mail address: mehdi.radnia@gmail.com 